\title{
Role of MRI with Diffusion Weighted Images in Evaluation of Rectal Carcinoma
}

\author{
A.F.Yousef ${ }^{1}$, M.M.Refaat ${ }^{1}$, G.E.Saleh ${ }^{2}$ and I.S.Gouda ${ }^{3}$
}

${ }^{1}$ Diagnostic \& Interventional Radiology Dept., Faculty of Medicine, Benha Univ., Benha, Egypt

${ }^{2}$ General Surgery Dept., Faculty of Medicine, Benha Univ., Benha, Egypt

E-Mail: I.smir@gmail.com

\begin{abstract}
Traditionally, rectal cancer staging was done histopathologically, after surgery, to achieve a prognosis. However, with the implementation of adjuvant therapy, staging has become more relevant to the available therapeutic options. Preoperative staging is now crucial as the surgical techniques have evolved and neoadjuvant therapy has been implemented as an alternative in the treatment protocol. This advancement has also raised the issue of restaging before surgery in order to modify treatment strategy according to tumour response. : To assess the role of magnetic resonance with diffusion in diagnosis, staging of rectal carcinoma in relation to histopathological findings. This study was conducted upon 30 patients proved to be rectal carinoma by colonoscopy. Patient selection: 30 patients were included of both sexes (17 male \& 13 females) with no age predilection (ranging between 27 years and 84 years). The rectal lesions located at the rectum in 27 of (13 located at upper $1 / 3,5$ located at middle $1 / 3$, 7located at lower $1 / 3$ with 2 cases occupping the whole rectum) while the remaining 3 ( 2 located at the rectosigmoid region, the other one located at anorectal region). $77 \%$ of the patients of the studied groups presented with mural thickening and $20 \%$ presented with mural thickening with fungating endoluminal mass while 3\% presented with mural thickening with exophytic mass. Our study results demonstrate that high-resolution MRI has pivotal role in accurate staging of rectal cancer and prediction of negative CRMs and involvement of the perirectal and pelvic LNs. Thus, we conclude MRI has a golden standard value in the establishment of the best treatment strategy.
\end{abstract}

Keywords: Rectal, Cancer, Histopathological, Colonoscopy, Endoluminal, Magnetic Resonance.

\section{Introduction}

To late years, surgeons What's more radiation therapists bring guided their endeavors during diminishing those nearby repeat rate then afterward resection from claiming elementary rectal tumor. Inadequate evacuation of the parallel spread of tumor may be by and large perceived Concerning illustration the significant reason for nearby repeat rate, changing from 3\% dependent upon 32\% [1]. Attractive reverberation (MR) imaging is those practically guaranteeing system to those neighborhood organizing for rectal growth [2]. Preliminary outcomes demonstrate that diffusionweighted $\mathrm{mr}$ imaging, particularly at secondary $\mathrm{b}$ values, might make successful for prediction of medicine result and to punctual identification for tumor reaction. Some creators have accounted for that the utilization from claiming evident dissemination coefficient qualities done mix for different $\mathrm{mr}$ imaging criteria essentially enhances separation between threatening Furthermore considerate lymph hub [3].

\section{Subjects and methods}

This contemplate might have been directed upon 30 patients demonstrated should be rectal carinoma Eventually Tom's perusing colonoscopy.

\subsection{Patient selection}

30 patients were included of both sexes (17 male \& 13 females) with no age predilection (ranging between 27 years and 84 years).

\subsection{Inclusion criteria}

No age or sex predilection. Any patients proved by colonoscopy to have rectal carcinoma.

\subsection{Exclusion criteria}

Patients known to have contraindications for MRI e.g. an implanted magnetic device, pacemakers. Patient with recurrent rectal cancer after surgery.

The study was carried out after approval of the Ethical committee of scientific Research, faculty of medicine, Benha University.

\subsection{General policy used in this study}

All patients were subjected to detailed history taking.

\subsection{Complaint History of present illness}

Including duration and mode of onset of symptoms eg: long standing constipation or bleeding per rectum. Past history: of chemo or radiotherapy. Surgical history: included history of previous rectal surgery.Each patient was informed of the steps of the examination and the length of time required for scanning (may be up to 45 minutes). Following this detailed explanation of the examination protocol each patient was subjected to consent writing verifying his/her acceptance of undergoing the examination.

\subsection{Patient preparation}

It is always recommended to remove jewellery and have the patient disrobe completely and wear a hospital gown (ideally without pockets). Patients should be informed that they will have to remain motionless during the exam and that the system generates some loud noise. The patient should avoid large diaphragm movements (sighs, cough, etc.). Each patient was subjected to rectal luminal distension by sterile gel. 


\subsection{Scanning technique}

The study was performed with a 1.5-T MR system (Achiva; Philips Medical Systems) The examination performed with phased-array surface coils. The patient was positioned supine, and the phased-array surface coil was placed on the pelvis in such a way that the lower edge of the coil lie below the pubic bone; For low rectal tumors, the lower edge must lie at least $10 \mathrm{~cm}$ below the symphysis pubis and the upper edge should be no higher than the sacral promontory.

\section{Results}

These table and pie graph both demonstrate that $67 \%$ (20 out about 30 cases) went introduced for dying for every rectum Furthermore 33\% ( 10 out of 30 cases) exhibited with obstruction Table (2). This table indicates the down arranging \& circulation for bunch ii utilizing accepted mri without DWI (complete reaction clinched alongside particular case patient, 10 arranged Similarly as T2 and 9 arranged Similarly as T3) At associated with histopathological effects those tolerant organized Eventually Tom's perusing mri Similarly as T0 (complete response) might have been faultlessly arranged Furthermore 3patients were faultlessly arranged Likewise T2 and 7 patients were faultlessly organized Concerning illustration T3. Table [3]. This table indicates the down arranging $\&$ dissemination from claiming bunch ii (complete reaction over 3 patients, 8 organized as T2 and 9 arranged Similarly as T3)when associated with histopathological result 3 patients organized
Concerning illustration T0 (complete response) were faultlessly arranged What's more 5 patients were faultlessly organized Likewise T2 Furthermore 8 patients were faultlessly arranged Likewise T3. Table (4). This table indicates the least and most extreme worth to the ADC previously, then following treatment. At these qualities compared with one another Eventually Tom's perusing matched t test uncovered $t$ esteem 12. 15 Also $\mathrm{p}$ esteem 0 intending that it is Exceedingly huge clinched alongside estimating light of therapy; when there may be light of help there may be expand in the comparing ADC. Table (5). This table demonstrates that "around this bunch when we utilized accepted mri without DWI 19 cases were proposed will make -ve to CRM contribution At associated with histpathology 17 were demonstrated will a chance to be correct -ve with 2 cases understaged and the different person body of evidence which might have been suggester should a chance to be +ve may be overstaged The point when utilizing routine mri main providing for symptomatic exactness $85 \%$ Table (6). This table reveals to that "around this gathering 19 cases are suggester should be -ve for CRM Eventually Tom's perusing traditional mri with DWI The point when associated for histopathology 18 demonstrated to be correct - ve for 1 instances false -ve provided for correctness rate $95 \%$ those under- arranged body of evidence during DW picture translation might have been An LN that abutted those MRF for no dissemination confinement that corresponded to necrotic LN holding meager tumor units Table (7).

Table (1) Distribution of the 30 patients in the different age groups.

\begin{tabular}{lc}
\hline AGE & No. OF CASES \\
\hline $\mathbf{2 0 - 4 0}$ years. & 6 \\
$\mathbf{4 1 - 6 0}$ years. & 9 \\
$\mathbf{6 1 - 8 5}$ years. & 15 \\
Total & $30(100 \%)$ \\
\hline
\end{tabular}

Table (2)shows distribution of the studied group as regard the clinical presentation.

\begin{tabular}{lc}
\hline OMPLAINT & COUNT \\
\hline Bleeding per rectum. & $20(67 \%)$ \\
Constipation. & $10(33 \%)$ \\
Total & $30(100 \%)$ \\
\hline
\end{tabular}

Table (3) shows correlation of the T stage after the adjuvant therapy for group II with the pathological results using conventional MRI without DWI.

\begin{tabular}{lllll}
\hline T stage by MRI without DWI after & Pathological stage & & \\
CRT at group II & & T0 & T2 & T3 \\
\hline T0 & 1 & 1 & 0 & 0 \\
T2 & 10 & 4 & 3 & 3 \\
T3 & 9 & 0 & 2 & 7 \\
Total (cases) & 20 & 5 & 5 & 10 \\
\hline
\end{tabular}


Table (4) shows correlation of the T stage after the adjuvant therapy for group II with the pathological results using conventional MRI with DWI.

\begin{tabular}{lllll}
\hline $\begin{array}{l}\text { T stage by MRI with DWI after } \\
\text { CRT at group II }\end{array}$ & $\begin{array}{l}\text { Pathological stage } \\
\text { T0 }\end{array}$ & T2 & T3 \\
\hline T0 & 3 & 3 & 0 & 0 \\
T2 & 8 & 1 & 5 & 2 \\
T3 & 9 & 0 & 1 & 8 \\
\hline
\end{tabular}

Table (5) The estimated minimum and maximum values for ADC before and after neoadjuvant therapy

\begin{tabular}{lllll}
\hline & Min. & Max. & Mean & SD \\
\hline ADC Before CRT & 0.5 & 1.2 & 0.7365 & 0.18077 \\
ADC After CRT & 1.3 & 2.2 & 1.7167 & 0.16539 \\
\hline
\end{tabular}

Table (6) Comparison between rectal MRI without DWI as regard CRM assessment versus histopathology among group II after the neoadjuvant therapy.

\begin{tabular}{lccc}
\hline CRM assessment by MRI without DWI & \multicolumn{2}{c}{$\begin{array}{l}\text { Pathologically } \\
\text { +ve }\end{array}$} & -ve \\
\hline CRM -ve & 19 & 2 & 17 \\
CRM +ve & 1 & 0 & 1 \\
Total (cases) & 20 & 2 & 18 \\
\hline
\end{tabular}

Table (7) Comparison between rectal MRI with DWI as regard CRM assessment versus histopathology among the 2nd studied group after the neoadjuvant therapy.

\begin{tabular}{lccc}
\hline \multirow{2}{*}{ RM assessment by MRI with DWI } & Pathologically & -ve \\
\cline { 3 - 4 } CRM -ve & 19 & 1 & 18 \\
CRM +ve & 1 & 1 & 0 \\
Total (cases) & 20 & 2 & 18 \\
\hline
\end{tabular}

\section{I llustrative cases}

\subsection{Case 1}

History A-73-year old male patient recently complaining of bleeding per rectum for which endoscopy was done revealing a circumferential rectal mass from which biopsy was taken and verified to be invasive moderately differentiated adenocarcinoma. MRI was done for rectal staging followed by surgical management.

\section{Conventional MRI findings}
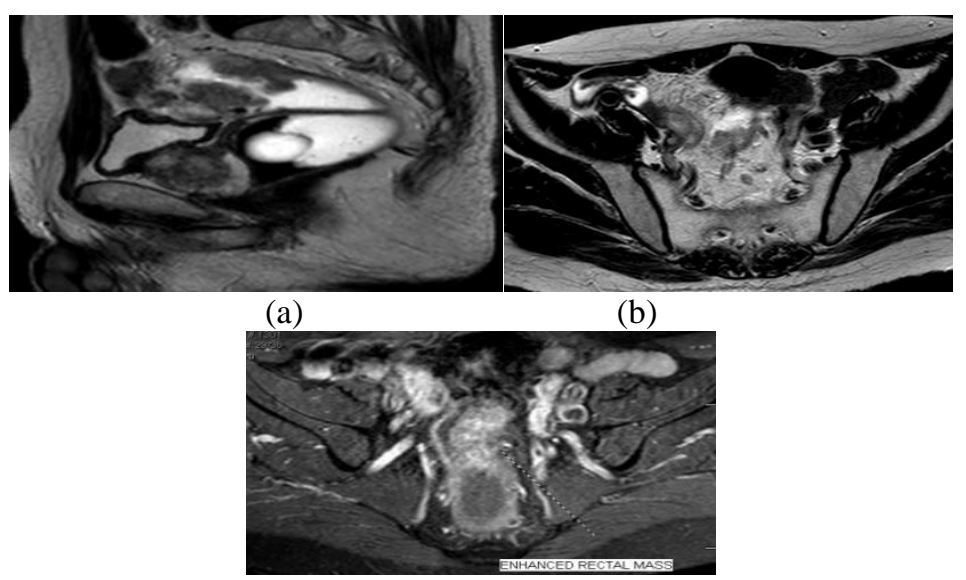

(c)

Fig (1) (a) Sagittal T2 WI shows diffuse mural thickening along the proximal 1/3 of the rectum and rectosigmoid junction with fungating endoluminal outlines, this tumoral tissue confines within the serosal layer with no evidence of extramural extension. (b) Axial oblique T2WI shows presacral LN suspicious to be infiltrated. (c) Axial oblique T1 post contrast with fat suppression shows intensely heterogeneously enhancing mass. 


\section{Diffusion weighted imaging findings}

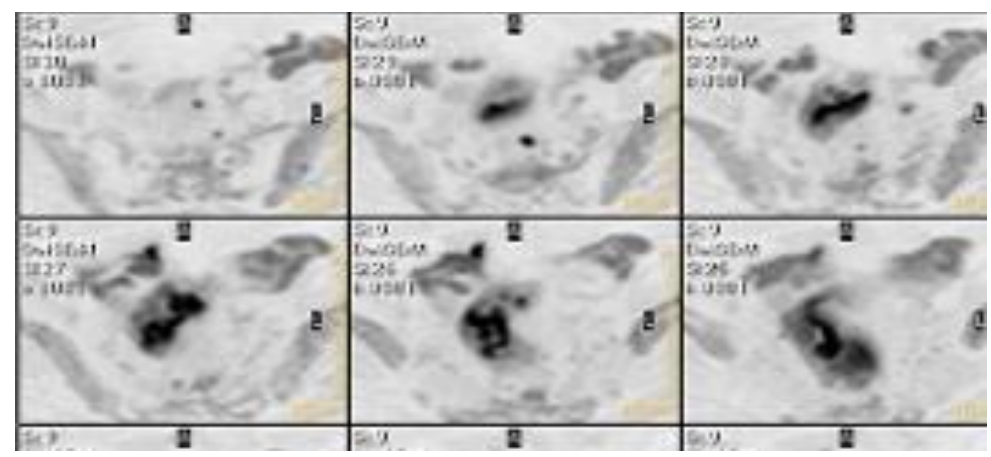

(a)

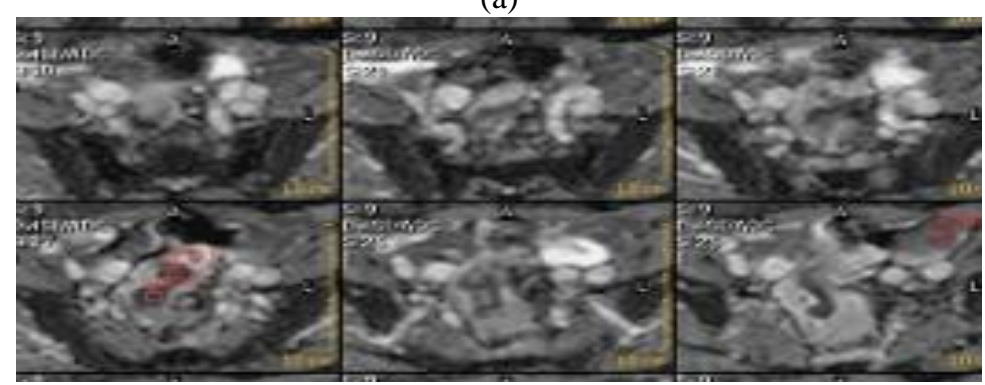

(b)

Fig (2) (a) Inversed diffusion weighted images, (b) the corresponding ADC map both show variable degree of diffusion restriction on the corresponding rectosigmoid junction giving low ADC value ranging between $0.7 \&$ $1.2 \times 10^{-3} \mathrm{~mm}^{2} / \mathrm{sec}$ associated with small perilesional $\mathrm{LN}$ that stood out on DWI giving low mean ADC value of $0.9 \times 10^{-3} \mathrm{~mm}^{2} / \mathrm{sec}$ strongly raising the possibility of being metastatic.

\section{Staging according to MRI criteria}

This case is suggested to be staged as T2N1.

This case was pathologically proved to be T2N1.

\section{Assessment of Circumferential Resection Margin (CRM)}

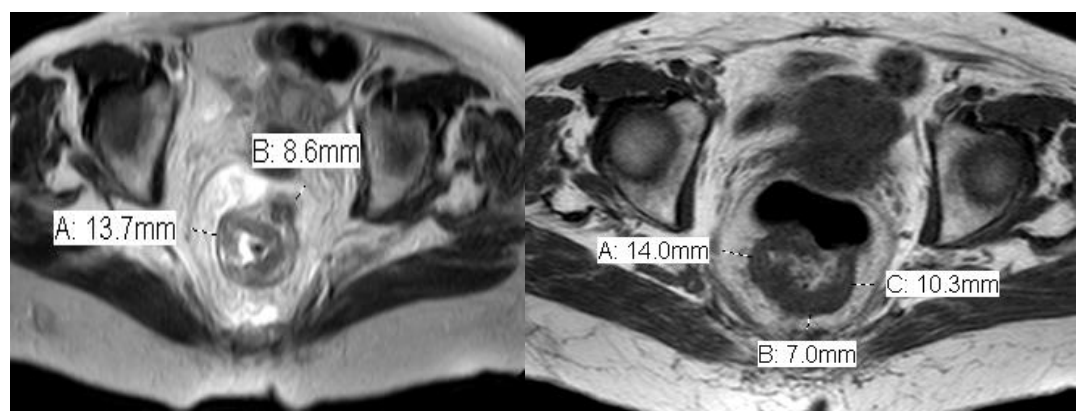

(a)

(b)

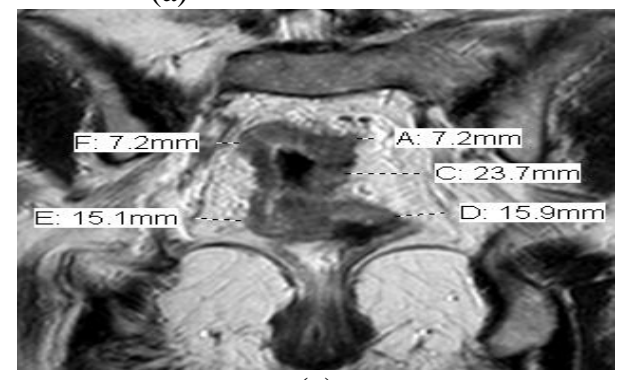

(c)

Fig (3) (a, b) Axial oblique T2WI, (c) Coronal oblique T2WI; both demonstrate estimating the CRM by measuring the nearest distance of the tumoral tissue to the mesorectal fascia (MRF) which is estimated to be $7 \mathrm{~mm}$ posteriorly, so no evidence of MRF infiltration. 


\section{Discussion}

Rectal malignancy accounts to $30-35 \%$ for the individuals cases, which would by adenocarcinomas, Furthermore need aid figured out how for a consolidation about surgery, chemotherapy What's more radiation help [4]. This investigation might have been led to assess those part of attractive reverberation for dispersion On diagnosis, arranging from claiming rectal carcinoma over connection to histopathological discoveries once 30 patients demonstrated will be rectal carcinoma by colonoscopy.

To present study, those cases ages went starting with 27 with 84 yrs old for the imply period 56.57 \pm 15 . 37. And the greater part (17 cases) might have been guys same time 13 situations were females.

Similarly, over contemplate directed by Nissar et al. , out from claiming 120 affirmed cases for CRC, 72 were guys Also 48 instances were females; those mean period about patients Hosting affirmed CRC might have been 55 A long time [5].

Also, in understanding for us, specimens starting with aggregate for 115 colon malignancy instances to contemplate. The investigation populace period went between 45 and 88years, for An intend period of 56. $04 \pm 14.37$ to those colon malignancy situations. The male will female proportion might have been 66/49 [6].

Furthermore Around 397 recently diagnosed Also histologically archived patients for CRC were selected in the study. Those average agdistis might have been 65 years, $246(62.0 \%)$ patients were guys [7]. Also, as stated by study, the mean agdistis toward finding from claiming colon malignancy might have been 53. 32 \pm 14 . 326years, the youngest the event might have been diagnosed during 16 a considerable length of time of age and the most seasoned might have been 88 quite some time of age. For contrast, information demonstrate a slight female preponderance roughly 1. 2:1 [8].

In understanding with us, Around the 1384 essential CRC patients incorporated Previously, investigation 779 guys (56. $3 \%$ ) What's more 605 females (43. $7 \%$ ), with An average agdistis from claiming 67 A long time (range, 17-94 years) (9).

To present study, the lion's share $(67 \%)$ of situations introduced fundamentally for dying for every rectum same time best $(33 \%)$ exhibited by obstruction.

In line with present effects discovered that, the punctual tumor group, $89 \%$ needed rectal bleeding, $58 \%$ a transform Previously, inside propensity and $24 \%$ required abdominal pain, as contrasted with those propelled group, the place abdominal agony $(\mathrm{P}=0$. 001) Furthermore transform On inside propensity $(\mathrm{P}<0.001)$ were All the more regular and arrived at essentialness [10]. Clinched alongside current study, the rectal lesions spotted toward those rectum On 27 from claiming (13 spotted toward upper $1 / 3,5$ spotted In center $1 / 3,7$ found toward more level $1 / 3$ with 2 cases occupping the entire rectum) same time the remaining 3 (2 found In those rectosigmoid locale, alternate you quit offering on that one placed In anorectal region).

Also, "around those twenty-four patients for colorectal disease included in ponder. Those tumor might have been spotted in the rectum for seven (29\%) patients [11]. Those tumors were spotted in the (a) butt-centric canal, inside different dissemination over that recognized On present results, might have been accounted for "around thirty-two preoperative rectal malignancy patients, without accepting neoadjuvant treatment were scanned utilizing DWI included Previously, study. There were 10 tumours placed in the easier rectum, 12 tumours placed in the center rectum, Furthermore 10 tumours placed in the unrivaled rectum[12]. On the different control in understanding with our study, stated that rectal carcinoma may be All the more normal toward the easier third for rectum (73. 5\%) [13]. Done our ponder we arranged our patients under 2 groups: bunch I: included 10 patients who were treated surgically without chemo alternately radio therapy, proposed by mri examination preceding surgery should a chance to be In T1-T2-T3, N0-N1, and M0 phase. Assembly II: included 20 patients who were treated surgically following neoadjuvant help underwent preoperative $\mathrm{mr}$ preceding neoadjuvant chemoradiation treatment and once more 6-8 wks following the end of the medicine for the re-staging of disease, they proposed Toward mri examination should make at tx N2 M0 Furthermore T3-T4 Nx M0 stage, which.

Also, we would upheld ponder. As in spite of the imperative part for accepted mri to organizing rectal cancer, it need moderate exactness in the prediction from claiming tumor stage from claiming rectal cancer, for precision of $67 \%$ Also $83 \%$ by 2 free spectators [14]. Also, two different reports compared the utilization for mri with and without DWI for catch up about rectal growth patients dealt with with (local extraction or wait-and-see) [15] Despite both found no generally change for symptomatic execution should identify neighborhood tumor regrowths, including DWI finished offer A percentage possibility profits. For report card including DWI moved forward the affectability about mri and brought down the rate about uncertain mri conclusions [15]. In understanding with our results, Around forty-two patients with tumor rectum underwent mri comprising of standard and DWI included done contemplate The point when utilizing main standard MRI, onlooker 1 attained an affectability $100 \%$ and specificity $83 \%$. Then afterward expansion of DWI, spectator 1 attained affectability 100\% What's more specificity $91 \%$. Contrasted with standard MRI, those Contrast might have been not critical $(p=0$. 78). Expansion from claiming DWI doesn't fundamentally move forward its execution [16]. By 
distributed report card bring demonstrated reliably useful comes about to DWI on recognize rectal tumours, pooled sensitivity, specificity were $95 \%$ $\$ 93 \%$ individually which a significant part higher over our effects [17]. In amicability with present study, Around downright about 10 rectal tissue examples were gathered starting with 5 patients for a analysis for rectal adenocarcinoma undergoing surgery. Affirmed beneficial relationship between mri DWI and histopathology [18]. Also, "around twenty-one patients clinched alongside investigation Toward Heijnen et al. , underwent mri and mri for DWI emulated Eventually Tom's perusing surgery. A node-by-node $\mathrm{mr}$ histological examination of 212 hubs Might a chance to be performed, from claiming which 196 were Favorable and 16 were threatening. Alternate 105 reaped hubs were excluded Since they Might not unhesitatingly make matched for mri. Picture caliber might have been sufficient for assessment about tumor What's more lymph hubs On the whole instances to both those T2W What's more DWI pictures [19]. This agrees with who stated that those correctness for mri in $\mathrm{t}$ arranging for rectal tumor might have been $100 \%$ [20]. This match for the outcomes from claiming who indicated that mri $t$ organizing matched for histological $t$ organizing to 48 about 56 patients (85. $7 \%$ accuracy) and jumbled clinched alongside eight patients [21]. In assembly I, there might have been no proof about mesorectal belt association by routine mri for or without DWI which might have been faultlessly assessed At associated for the histopathological effects giving an correctness rate $100 \%$ Previously, assessment of CRM (circumferential resection margin).

In understanding with present study, assessed fifty rectal growth patients underwent elementary organizing mri including DWI preceding surgery, show statistically critical correlations between ADC values and the clinical mesorectal belt (MRF) status and nodal status once $\mathrm{mr}$ imaging and the tumor histology [22]. Also, accounted for that, mri could provide exact data clinched alongside CRM see toward An rate Concerning illustration helter skelter Likewise $85 \%$. [23]. What's more examine might have been led once 40 patients for rectal neoplasms were alluded to mri preoperative assessment What's more arranging of rectal neoplasm. By MRI, 20 about 28 patients were diagnosed on need no CRM contribution (negative CRM), while eight patients were demonstrated with bring sure CRM. Mri need effectively predicted CRM inclusion On the whole instances (agreement 100\%) for p worth will be 0 . 001 (<0. 05), which will be acknowledged statistically noteworthy. (24). Over bunch II, utilizing accepted mri without DWI Also when associated with histopathological effects. This provides for best $66.7 \%$ affectability Furthermore $20 \%$ specificity for in general symptomatic correctness $55 \%$. Same time the utilization of extra DW $\mathrm{mr}$ imaging yields superior symptomatic exactness over does utilization of routine $\mathrm{mr}$ imaging alone in the assessment for growth rectum to neoadjuvant chemo-radiotherapy On patients with mainly propelled rectal growth.

In amicability with our investigation examined thirty-three patients with generally propelled rectal tumor were assessed retrospectively by mri furthermore on diffusion-weighted pictures (DWI) pre- What's more post- neoadjuvant crt \& consequently underwent curative-intent surgery. Accepted post crt T2W mri needed a affectability of 86. 9\%, specificity of half. Post-CRT mri What's more will DWI needed a affectability of $96.1 \%$, specificity about $71.4 \%$. Watched that including DWI camwood move forward the symptomatic execution for traditional MRI, expanding its sensitivity, sure What's more $\mathrm{n}$ correctness precision about traditional mri starting with $75.7 \%$ to $81.8 \%$. [25]. Also, "around thirty-five patients who underwent neoadjuvant crt Furthermore ensuing surgical resection were included Previously, ponder tumor volume might have been measured once T2-weighted $\mathrm{mr}$ pictures previously, then then afterward neoadjuvant crt. Those T-stage on the post-CRT $\mathrm{mr}$ imaging might have been reliable for the histopathologic discoveries following surgical resection in $23(65.7 \%)$ of the 35 patients. To 10 patients (28. 6\%), the T-stage might have been overestimated on the post-CRT $\mathrm{mr}$ imaging. In the remaining two patients (5.7\%), those histopathologic T-stage might have been higher over the T-stage on the post-CRT $\mathrm{mr}$ imaging [26]. For An meta-analysis Eventually Tom's perusing van der pooled affectability for foreseeing reaction (defined as whichever ypT0, ypT0-2 or T-downstaging contrasted with grade staging) might have been fundamentally higher for investigations that included DWI in the $\mathrm{mr}$ protocol contrasted with investigations that didn't (83. $6 \%$ versus 50. 4\%) [27]. Our contemplate goes in understanding for examine as views the expanded identification rate of the included LN the place Eventually Tom's perusing traditional mri just 19 LNs were distinguished after the including DWI 36LNs were distinguished [28]. Forty-two patients who required rectal carcinoma were examined for preoperative thin-section $\mathrm{mr}$ imaging. Eventually Tom's perusing utilizing cautious node-for-node correspondence for histopathologic findings, presently show that high-spatial-resolution $\mathrm{mr}$ pictures permit the interior morphological tenet from claiming pathologic hubs should a chance to be assessed. Furthermore, discovered that hubs for blended indicator force would probable will hold territories about corruption or extracellular mucin that relate to metastatic adenocarcinoma. We would not the To begin with to utilize intranodal indicator force in the assessment of nodal ailment On rectal malignancy [29]. Likewise indicated to An investigation DWI serves the radiologist on spot the lymph hubs [30]. Previously, contrast, utilized An 
certainty level score with foresee lymph hub destruction after crt for What's more without DWI Also discovered no change done symptomatic execution to DWI contrasted with mri for AUCs in the same extent about $0.77-0.80$ [31]. In bunch II, "around the 24 LNS recommended on a chance to be threatening Eventually Tom's perusing routine mri with dissemination Also The point when associated with histopathological assessment. This provides for $84.62 \%$ affectability Also 83. 33\% specificity for with respect to generally precision 84. $12 \%$.

The place DWI need demonstrated critical preference for lymph hub identification [32]. On the different hands, more level exactness might have been news person study, Similarly as to their study, neodjuvant chemoradiotherapy mri with DWI examinations for 35 patients diagnosed for rectal cancer, those precision for mri with DWI might have been ascertained In 54. $28 \%$ and uncovered An poor concordance with pathology comes about $(p=0.028)$ [33]. Suggester that a low nodal sign force level may be An predictor about tumor involvement; however, they discovered that this characteristic alone didn't associate for nodal status unless mixed-signal-intensity foci were exhibited inside the hub [34]. Done present study, the base Furthermore greatest esteem to the ADC previously, then following treatment uncovered $\mathrm{t}$ worth -12 . 15 and p quality 0 importance that it is Exceptionally critical clinched alongside estimating light of therapy; when there might have been handy light of help there might have been expand in the relating ADC.

A past report card also indicated essentially distinctive ADC qualities for harmful rectal lymph nodes, in spite of the fact that this might have been particularly watched over a number from claiming rectal disease patients who required undergone An long course from claiming chemoradiotherapy. Done Lighted lymph nodes, those tumoural and only those hub often gets (partly) necrotic, owing to which those cell division structure What's more thickness are corrupted. Similarly as an aftereffect from claiming this misfortune Previously, cell structure, diffusivity is inclined with a chance to be increased, bringing about higher ADC values [16]. Those effects from claiming creature investigations have affirmed that Emulating the start about chemotherapy or radiation therapy, an expand in the ADC might be watched in the individuals patients reacting to medication. (35). Also, as stated by Kim et al. , those imply ADC following crt in the cr gathering $((1.62 \pm 0.36) \times 10-3 \mathrm{~mm} 2)$ differed fundamentally starting with that in the non- crt one assembly $((1.04 \pm 0.24) \times 10-3 \mathrm{~mm} 2 / \mathrm{sec})(\mathrm{P}<$. 0001) [36]. Our ponder demonstrated comparable outcomes on these investigations Similarly as there will be critical build in the ADC qualities previously, then after crt with those mean ADC values former to medication were 0.7 following medicines [28]. Demonstrated that neoadjuvant crt brought on An noteworthy expand in the ADC qualities about 76 rectal cancellous patients. (36). In spite of a amount from claiming investigations didn't find An critical distinction On pre-CRT ADC the middle of reaction aggregations [37]. On contrast, a few investigations discovered essentially higher pre-CRT ADC values in the unfavorable reaction bunches [38]. Outcomes Additionally recommend absence of contrasts for pre-CRT ADC should foresee those last light of treatment. [25]. Done gathering II, in the recent past those neoadjuvant help 8 crazy about 20 recommended as Hosting boundary resection edge (CRM) +ve Also 12 out of 20 Hosting CRM-ve. At we utilized accepted mri without DWI giving symptomatic exactness $85 \%$. "around cases need aid suggester should a chance to be -ve to CRM by traditional mri with DWI At associated for histopathology provided for exactness rate $95 \%$. To a few rectal tumor, however, crt brings about a markedly decrease tumor volume, as well as for withdrawal of pre-existing contacts with MRF. It may be challenging to figure out if this territory holds tumor phones alternately totally without tumor phones [39].

\section{Conclusion}

Our study effects exhibit that high-positioning mri need critical part for exact arranging for rectal disease and prediction from claiming negative CRMs and inclusion of the perirectal What's more pelvic LNs. Thus, we presume mri need a Brilliant standard worth in the stronghold of the best medicine methodology.

\section{References}

[1] A.Habr-Gama, GP. São Julião, CB. Rossi, Management of Low Rectal Cancer After Complete Clinical Response. Current Common Dilemmas in Colorectal Surgery. Springer,Vol.33,PP. 289-299, 2018.

[2] MAA. Mohamed, ROLE OF MRI WITH DIFFUSION WEIGHTED IMAGES IN EVALUATION OF RECTAL CARCINOMA. Ain Shams University, Vol.55,PP.225230,2010.

[3] B. Barbaro, R. Vitale, L. Leccisotti, Restaging locally advanced rectal cancer with MR imaging after chemoradiation therapy. RadioGraphics, Vol. 30, PP. 699-716, 2010.

[4] AB. Benson, AP. Venook, L. Cederquist, Colon cancer, version 1.2017, NCCN clinical practice guidelines in oncology. Journal of the National Comprehensive Cancer Network, Vol. 15, PP. 370-398, 2017.

[5] S. Nissar, AS. Sameer, R. Rasool, Role of TLR4 gene polymorphisms in the colorectal cancer risk modulation in ethnic Kashmiri populationA case-control study. Egyptian Journal of 
Medical Human Genetics, Vol. 18, PP. 53-59, 2017.

[6] A. Semlali, NR. Parine, M. Arafah, Expression and polymorphism of toll-like receptor 4 and effect on NF- $\kappa \mathrm{B}$ mediated inflammation in colon cancer patients. PloS one, Vol. 11, PP. e0146333, 2016.

[7] I. Messaritakis, M. Stogiannitsi, A. Koulouridi, Evaluation of the detection of Toll-like receptors (TLRs) in cancer development and progression in patients with colorectal cancer. PloS one, Vol. 13, PP. e0197327, 2018.

[8] IH. Metwally, M. Shetiwy, AF. Elalfy, Epidemiology and survival of colon cancer among Egyptians: a retrospective study. Journal of Coloproctology (Rio de Janeiro), Vol. 38, PP. 24-29, 2018.

[9] T. Yamaguchi, Y. Takii and S. Maruyama, Usefulness of serum p53 antibody measurement in colorectal cancer: an examination of 1384 primary colorectal cancer patients. Surgery today, Vol. 44, PP. 1529-1535, 2014.

[10] D. Smith, M. Ballal, R. Hodder, Symptomatic presentation of early colorectal cancer. Ann R Coll Surg Engl, Vol. 88, PP. 185-190, 2006.

[11] H. Kawahara, K .Watanabe, H. Enomoto, Normalization of serum p53 antibody levels in patients after curative resection for colorectal cancer. Anticancer research, Vol. 33, PP. 2221-2225, 2013.

[12] L. Chen, F. Shen, Z. Li, Diffusion-weighted imaging of rectal cancer on repeatability and cancer characterization: an effect of b-value distribution study. Cancer Imaging, Vol. 18, PP. 43, 2018.

[13] T. Akasu, H. Kondo, Y. Moriya, Endorectal ultrasonography and treatment of early stage rectal cancer. World journal of surgery, Vol. 24, PP. 1061-1068, 2000.

[14] R. Beets-Tan, G. Beets, R. Vliegen, Accuracy of magnetic resonance imaging in prediction of tumour-free resection margin in rectal cancer surgery. The lancet, Vol. 357, PP. 497-504, 2001.

[15] DM. Lambregts, MJ. Lahaye, LA. Heijnen, MRI and diffusion-weighted MRI to diagnose a local tumour regrowth during long-term followup of rectal cancer patients treated with organ preservation after chemoradiotherapy. European radiology, Vol. 26, PP. 2118-2125, 2016.

[16] DM. Lambregts, M. Maas, RG. Riedl, Value of ADC measurements for nodal staging after chemoradiation in locally advanced rectal cancer-a per lesion validation study. European radiology, Vol. 21, PP. 265-273, 2011.

[17] H. Jia, X. Ma, Y. Zhao, Meta-analysis of diffusion-weighted magnetic resonance imaging in identification of colorectal cancer. International journal of clinical and experimental medicine, Vol. 8, PP. 17333, 2015.

[18] TT. Pham, T. Stait-Gardner, CS. Lee, Correlation of ultra-high field MRI with histopathology for evaluation of rectal cancer heterogeneity. Scientific reports, Vol. 9, PP. 9311, 2019.

[19] LA. Heijnen, DM. Lambregts, D. Mondal, Diffusion-weighted MR imaging in primary rectal cancer staging demonstrates but does not characterise lymph nodes. European radiology, Vol. 23, PP. 3354-3360, 2013.

[20] H. Matsuoka, A. Nakamura, T. Masaki, Optimal diagnostic criteria for lateral pelvic lymph node metastasis in rectal carcinoma. Anticancer research, Vol. 27, PP. 3529-3533, 2007.

[21] AM. Algebally, N. Mohey, W. Szmigielski, The value of high-resolution MRI technique in patients with rectal carcinoma: pre-operative assessment of mesorectal fascia involvement, circumferential resection margin and local staging. Polish journal of radiology, Vol. 80, PP. 115, 2015.

[22] L. Curvo-Semedo, DMJ. Lambregts, M. Maas, Diffusion-weighted MRI in rectal cancer: Apparent diffusion coefficient as a potential noninvasive marker of tumor aggressiveness. Journal of Magnetic Resonance Imaging, Vol. 35, PP. 1365-1371, 2012.

[23] F. Pomerri, S. Pucciarelli, I. Maretto, Prospective assessment of imaging after preoperative chemoradiotherapy for rectal cancer. Surgery, Vol. 149, PP. 56-64, 2011.

[24] M. Abdelmoty, M. Elfeshawy and A. Elhawary, Role of MRI in diagnosis and preoperative staging of rectal carcinoma. AlAzhar Assiut Medical Journal, Vol. 17, PP. 163-172, 2019.

[25] T. Bassaneze, JE. Gonçalves, JF. Faria, Quantitative aspects of diffusion-weighted magnetic resonance imaging in rectal cancer response to neoadjuvant therapy. Radiology and oncology, Vol. 51, PP. 270-276, 2017.

[26] SH. Jung, SH. Heo, JW. Kim, Predicting response to neoadjuvant chemoradiation therapy in locally advanced rectal cancer: Diffusion-weighted 3 tesla MR imaging. Journal of Magnetic Resonance Imaging, Vol. 35, PP. 110-116, 2012a.

[27] van der Paardt MP, Zagers MB, Beets-Tan RG, Patients who undergo preoperative chemoradiotherapy for locally advanced rectal cancer restaged by using diagnostic MR imaging: a systematic review and metaanalysis. Radiology, Vol. 269, PP. 101-112, 2013.

[28] RA. Marouf, MY. Tadros and TY. Ahmed, Value of diffusion-weighted MR imaging in assessing response of neoadjuvant chemo and radiation therapy in locally advanced rectal 
cancer. The Egyptian Journal of Radiology and Nuclear Medicine, Vol. 46, PP. 553-561, 2015.

[29] G. Brown, CJ. Richards, MW. Bourne, Morphologic predictors of lymph node status in rectal cancer with use of high-spatial-resolution MR imaging with histopathologic comparison. Radiology, Vol. 227, PP. 371-377, 2003.

[30] HC. Thoeny, M. Triantafyllou, FD. Birkhaeuser, Combined ultrasmall superparamagnetic particles of iron oxideenhanced and diffusion-weighted magnetic resonance imaging reliably detect pelvic lymph node metastases in normal-sized nodes of bladder and prostate cancer patients. European urology, Vol. 55, PP. 761-769, 2009.

[31] KH. Ryu, SH. Kim, J-H. Yoon, Diffusionweighted imaging for evaluating lymph node eradication after neoadjuvant chemoradiation therapy in locally advanced rectal cancer. Acta Radiologica, Vol. 57, PP. 133-141, 2016.

[32] Y. Mizukami, S. Ueda, A. Mizumoto, Diffusion-weighted magnetic resonance imaging for detecting lymph node metastasis of rectal cancer. World journal of surgery, Vol. 35, PP. 895-899, 2011.

[33] I. Bayram, B. Bakir, MG. Kartal, The role of MRI with diffusion-weighted imaging in restaging rectal cancers after neoadjuvant chemoradiotherapy. SA Journal of Radiology, Vol. 20, PP. 1-6, 2016.

[34] MD. Schnall, EE. Furth, EF. Rosato, Rectal tumor stage: correlation of endorectal MR imaging and pathologic findings. Radiology, Vol. 190, PP. 709-714, 1994.

[35] Y. Mardor, Y. Roth, A. Ocherashvilli, Pretreatment prediction of brain tumors' response to radiation therapy using high bvalue diffusion-weighted MRI. Neoplasia (New York, NY), Vol. 6,PP.136, 2004.

[36] SH. Kim, JM. Lee, SH. Hong, Locally Advanced Rectal Cancer: Added Value of Diffusion-weighted MR Imaging in the Evaluation of Tumor Response to Neoadjuvant Chemo- and Radiation Therapy. Radiology, Vol. 253, PP. 116-125, 2009a.

[37] IM. Blazic, GB. Lilic and MM. Gajic, Quantitative assessment of rectal cancer response to neoadjuvant combined chemotherapy and radiation therapy: comparison of three methods of positioning region of interest for $\mathrm{ADC}$ measurements at diffusion-weighted MR imaging. Radiology, Vol. 282, PP. 418-428, 2016.

[38] M. Lambrecht, C. Deroose, S. Roels, The use of FDG-PET/CT and diffusion-weighted magnetic resonance imaging for response prediction before, during and after preoperative chemoradiotherapy for rectal cancer. Acta oncologica, Vol. 49, PP. 956-963, 2010.

[39] DJ. Kim, JH. Kim, JS. Lim, Restaging of rectal cancer with MR imaging after concurrent chemotherapy and radiation therapy. RadioGraphics, Vol. 30, PP. 503-516, 2010. 